\title{
Prevalence and Antibiotics Susceptibility Pattern of Salmonella and Shigella Species among Diarrheal Patients Attending Nekemte Referral Hospital, Oromia, Ethiopia
}

\author{
Alemayehu Terfassa ${ }^{1}$ and Mulissa Jida $\mathbb{D}^{1,2}$ \\ ${ }^{1}$ Department of Biology, College of Natural and Computational Sciences, Wollega University, Nekemte, Ethiopia \\ ${ }^{2}$ Environmental Biotechnology Directorate, Ethiopian Biotechnology Institute, Addis Ababa, Ethiopia \\ Correspondence should be addressed to Mulissa Jida; mulaeabageda@gmail.com
}

Received 25 July 2017; Revised 3 December 2017; Accepted 28 December 2017; Published 24 January 2018

Academic Editor: Joseph Falkinham

Copyright (C) 2018 Alemayehu Terfassa and Mulissa Jida. This is an open access article distributed under the Creative Commons Attribution License, which permits unrestricted use, distribution, and reproduction in any medium, provided the original work is properly cited.

\begin{abstract}
The main objective of this study was determining the prevalence and antibiotics resistance pattern of Salmonella and Shigella sp. from diarrheal patients attending Nekemte Referral Hospital. A total of 422 patients were included in the study and their sociodemographic and clinical information was collected using questionnaire. Stool samples of the patients were collected and processed following standard bacteriological protocols. Presumptive colonies of Salmonella and Shigella species were identified and subcultured to their respective genera by standard biochemical tests. Antibiotics susceptibility of the isolates was tested using disk diffusion assay. The prevalences of Salmonella and Shigella sp. among the patients were $7.1 \%$ and $2.1 \%$, respectively. The antimicrobial susceptibility test results of the isolates showed that they are highly resistant to amoxicillin $(30 \mu \mathrm{g})$. In contrast, the isolates showed relatively lower resistance level to ceftriaxone $(30 \mu \mathrm{g})$, nalidixic acid $(30 \mu \mathrm{g})$, norfloxacin $(10 \mu \mathrm{g})$, and ciprofloxacin $(5 \mu \mathrm{g})$. This study revealed 9.2\% prevalence of Salmonella and Shigella sp. which were resistant to commonly prescribed antibiotics. Thus, intervention measures such as health education, provision of safe drinking water, improvement of waste disposal systems, and surveillance of antibiotics susceptibility of the pathogens should be done regularly.
\end{abstract}

\section{Introduction}

Acute gastroenteritis is one of the leading causes of illness and death in infants, children, immune-compromised, and aged individuals throughout the world. Of the enteric pathogens Salmonella and Shigella species are of particular concerns as causes of enteric fevers, food poisoning, and gastroenteritis $[1,2]$. They are transferred from person to person usually by asymptomatic carriers and via contaminated food and water. They are Gram-negative rods which commonly inhabit intestinal tracts of humans and many animals [3-5]. Currently, Salmonella and Shigella species are progressively becoming resistant to the commonly used antimicrobial agents [6-9]. The emergence of antibiotic resistance among their species is serious problem in developing countries.

Diseases caused by Salmonella and Shigella species are common public health problems in many parts of the world, including Ethiopia [10-13]. Studies conducted in different cities of Ethiopia indicated that they are widespread among diarrheal patients [8, 14-16]. Moreover, their increased resistance to commonly used antimicrobials has also been noted [13-16]. On the contrary, there is no information on their prevalence and antimicrobial resistance patterns in Nekemte. Therefore, this study was aimed at determining prevalence and antimicrobial resistance patterns of Salmonella and Shigella species among diarrheal patients attending Nekemte Referral Hospital.

\section{Materials and Methods}

2.1. Study Area, Design and Population. This study was conducted in Nekemte Referral Hospital from October 2015 to February 2016. Nekemte is located in Western parts of Oromia regional state at geographical location of $9^{\circ} 46^{\prime} \mathrm{N}$ 
latitude and $36^{\circ} 31^{\prime} \mathrm{E}$ longitude, with elevation ranging from $1,960 \mathrm{~m}$ to $2,170 \mathrm{~m}$ a.s.l. The study populations were all patients with acute diarrhea attending Nekemte Referral Hospital. Patients who were under antibiotics treatment in the last 14 days of sampling period were excluded from the study.

A cross-sectional study design was carried out to determine the prevalence and antimicrobial resistance pattern of Salmonella and Shigella species among diarrheal patients attending Nekemte Referral Hospital. Pretested questionnaire was used to gather information concerning sociodemographic data, clinical characteristics, and hygiene practice of the patients.

2.2. Sampling and Sample Size Determination. The study participants were selected via systematic random sampling technique from OPD of the hospital. A minimum sample size of 384 was calculated using single population proportion formula, assuming 95\% confidence interval, 50\% prevalence, and 5\% marginal error; as a result, 422 patients were included in the study [17].

2.3. Stool Sample Collection and Handling. Stool specimens were collected in sterile container and transported to Microbiology Laboratory, Oromia Regional Health Laboratory, Nekemte, within 2 hrs of collection. All plates and test tubes were incubated at $37^{\circ} \mathrm{C}$ for $24 \mathrm{hrs}$.

2.4. Isolation of Salmonella and Shigella Species. About $1 \mathrm{~g}$ of the stool sample was transferred to Selenite F broth and incubated at $37^{\circ} \mathrm{C}$ for $24 \mathrm{hrs}$. Simultaneously, a loop full of stool sample suspension was streaked on SSA and XLDA and then incubated at $37^{\circ} \mathrm{C}$ for $24 \mathrm{hrs}$. Furthermore, culture negative specimens on SSA and XLDA media were subcultured from Selenite F broth enrichment broth to SSA and XLDA plates to improve recovery of the isolates. Growth of Salmonella and Shigella species were detected by their typical colonies characteristic on SSA and on XLDA [18]. All isolates were purified by repeated subculturing, streaked on nutrient agar slant and preserved in refrigerator set at $4^{\circ} \mathrm{C}$ until further analysis.

\subsection{Morphological and Biochemical Characterization of the Isolates}

2.5.1. Morphological Characterization. Purified single colony from SSA/XLDA was taken with loop, spread on the center of clean slide, and subject to Gram staining. Finally, the stained smear was observed under microscope (oil immersion objective) and then morphology, cell arrangement, and Gram reaction of the isolates were determined.

2.5.2. Motility Test. To determine whether the tested organism was motile or not, motility media were inoculated with a straight inoculating needle through a single stab about $1-2 \mathrm{~cm}$ down into the medium and incubated at $37^{\circ} \mathrm{C}$ for $24 \mathrm{hrs}$. Based on their appearance on motility medium the test organisms were categorized as motile and nonmotile.
2.5.3. Biochemical Characterization of the Isolates. Colonies with typical characteristics of Salmonella and Shigella species from primary media were further cultured on Kligler Iron Agar (KIA) medium by stabbing the butt and streaking the slant and then incubated at $37^{\circ} \mathrm{C}$ for $24 \mathrm{hrs}$ to determine their glucose and lactose fermentation abilities and production of hydrogen sulfide [19]. The KIA tubes were examined for specific growth and appearance of Salmonella and Shigella species. Colonies of the isolates from primary media were further examined by stabbing the butt and streaking on surface of the slant of Lysine Iron Agar (LIA) and incubating at $37^{\circ} \mathrm{C}$ for $24 \mathrm{hrs}$. The growth of positive isolate was detected by their specific growth and appearance on LIA tubes [19]. Pure colonies of the isolates were tested for citrate utilization ability by stabbing the butt and streaking the slant of Simmons Citrate Agar and incubating at $37^{\circ} \mathrm{C}$ for $24 \mathrm{hrs}$ [19]. Suspected colonies of Salmonella and Shigella species were transferred from growth of KIA to nutrient broth with sterile loop and incubated for few hrs. Indole productions were tested by adding 5 drop of Kovac's reagent into the test tubes. Urease production ability of the suspected colony of Salmonella and Shigella was tested by inoculating heavily over the entire surface of the slant urease medium; the caps were loosened and incubated for an overnight at $37^{\circ} \mathrm{C}[19]$.

2.5.4. Antibiotics Susceptibility Test. Antibiotics resistance pattern of the isolates was determined using disc diffusion methods [20]. A single pure colony of each isolates was inoculated into the nutrient broth and incubated at $37^{\circ} \mathrm{C}$ for $24 \mathrm{hrs}$ and their turbidity was adjusted with $0.5 \mathrm{McF}$ arland. The culture was inoculated on Muller Hinton agar using sterilized cotton swab. Discs impregnated with appropriate concentration of antibiotics were placed on inoculated plate. Amoxicillin $(30 \mu \mathrm{g})$, ceftriaxone $(30 \mu \mathrm{g})$, chloramphenicol $(30 \mu \mathrm{g})$, ciprofloxacin $(5 \mu \mathrm{g})$, gentamicin $(10 \mu \mathrm{g})$, nalidixic acid $(30 \mu \mathrm{g})$, and norfloxacin $(10 \mu \mathrm{g})$ disks were used for the test. E. coli (ATCC 25922) was used as reference strain to ensure quality of the data. Zones of inhibition were measured to the nearest $\mathrm{mm}$ and results were interpreted according to the CLSI [20].

2.6. Data Analysis and Interpretation. The data was edited and analyzed using SPSS version 23. Multivariate logistic regression test, adjusted odds ratio (AOR), and 95\% CI $(P<$ 0.05 significance level) were used to assess the level of association among prevalence of the pathogens and associated risk factors.

\section{Results}

3.1. Sociodemographic and Clinical Characteristics of the Patients. In this study, 422 patients with acute diarrhea and attending Nekemte Referral Hospital from October 2015 to February 2016 were considered. Of these $52.8 \%$ were male and the mean age of the study participants was 20.7 years (Table 1$)$. The majority of study participants (83.2\%) were urban dwellers and $62.1 \%$ of them have the habit of washing their hands regularly after using toilet. Most (83.2\%) of the 
TABLE 1: Sociodemographic, clinical characteristics, and prevalence of Salmonella and Shigella sp. among diarrheal patients attending Nekemte Referral Hospital, October 2015 to February 2016.

\begin{tabular}{|c|c|c|c|}
\hline Variables & Frequency & Percent (\%) & Number of Salmonella or Shigella species positive patients (\%) \\
\hline \multicolumn{4}{|l|}{ Sex } \\
\hline M & 223 & 52.8 & $17(7.6)$ \\
\hline $\mathrm{F}$ & 199 & 199 & $22(11.1)$ \\
\hline \multicolumn{4}{|l|}{ Age Category (years) } \\
\hline $1-19$ & 225 & 53.3 & $18(8)$ \\
\hline $19-29$ & 81 & 19.2 & $6(7.4)$ \\
\hline $30-44$ & 69 & 16.4 & $11(15.9)$ \\
\hline $45-59$ & 35 & 8.3 & $4(11.4)$ \\
\hline$>60$ & 12 & 2.8 & 0 \\
\hline \multicolumn{4}{|l|}{ Educational status } \\
\hline Illiterate & 137 & 32.5 & $12(8.8)$ \\
\hline Grade $1-4$ & 70 & 16.6 & $4(5.3)$ \\
\hline Grade 5-8 & 71 & 16.8 & $4(5.6)$ \\
\hline Grade 9-12 & 81 & 19.2 & $11(13.6)$ \\
\hline Certificate dip. & 57 & 13.5 & $7(12.3)$ \\
\hline 1st degree \& above & 6 & 1.4 & $1(16.7)$ \\
\hline \multicolumn{4}{|c|}{ Hand washing after toilet } \\
\hline Good & 262 & 62.1 & $29(11.1)$ \\
\hline Poor & 160 & 37.9 & $10(6.3)$ \\
\hline \multicolumn{4}{|c|}{ Source of drinking water } \\
\hline Tap & 351 & 83.2 & $31(8.8)$ \\
\hline River & 70 & 16.6 & $8(11.4)$ \\
\hline well & 1 & 0.2 & $0(0.0)$ \\
\hline \multicolumn{4}{|l|}{ Residence areas } \\
\hline Urban & 320 & 91.1 & $31(8.8)$ \\
\hline Rural & 63 & 8.9 & $8(11.3)$ \\
\hline \multicolumn{4}{|l|}{ Clinical characteristics } \\
\hline Abdominal pain & 419 & 99.3 & $38(9.1)$ \\
\hline Vomiting & 272 & 64.5 & $28(10.3)$ \\
\hline Fever & 310 & 73.5 & $38(12.3)$ \\
\hline Headache & 222 & 52.6 & $34(15.3)$ \\
\hline Joint pain & 182 & 43.1 & $27(14.8)$ \\
\hline \multicolumn{4}{|c|}{ Prevalence of Salmonella \& Shigella sp. } \\
\hline Shigella sp. & 9 & 2.1 & - \\
\hline Salmonella sp. & 30 & 7.1 & - \\
\hline Total Prevalence & 39 & 9.2 & - \\
\hline
\end{tabular}

Note. Salmonella and Shigella positive values as No (\%), sp.: species.

diarrheal patients sources of drinking water was tap whereas $16.6 \%$ of them depend on river. Abdominal pain, fever, vomiting, and headache were the clinical symptoms observed among the study population. Abdominal pain was found to be the most common (99.3\%) clinical symptom followed by fever and vomiting (Table 1).

3.2. Prevalence of Salmonella and Shigella Species. Out of the total stool specimens, 9.2\% were positive for Salmonella and Shigella species (Table 1). Of these, $7.1 \%$ and $2.1 \%$ were positive for Salmonella and Shigella species, respectively. $77.7 \%$ of
Shigella isolates were obtained from male patients. Of all isolates, $46.1 \%$ were isolated from patients with age less than 19 years. In contrast, neither Salmonella nor Shigella species were found in study participants with age of 60 years and above. Our results also indicated that there were no statistically significant associations $(P>0.05)$ between gender, age groups, and prevalence of Salmonella and Shigella species, whereas poor hand washing habit after toilet (AOR: 4.1, $P: 0.002$ ), vomiting (AOR: 10.0, P: 0.040), and headache (AOR: $4.7, P: 0.013)$ had statistically significant $(P<0.05)$ association with the rate of isolation of the pathogens (data not shown). 
TABLe 2: Antibiotics susceptibility pattern of Salmonella and Shigella species from diarrheal patients attending Nekemte Referral Hospital, October 2015 to February 2016.

\begin{tabular}{|c|c|c|c|c|c|c|}
\hline \multirow{2}{*}{ Antimicrobial } & \multicolumn{3}{|c|}{ Salmonella species $N(\%)$} & \multicolumn{3}{|c|}{ Shigella species $N(\%)$} \\
\hline & S & I & $\mathrm{R}$ & S & I & $\mathrm{R}$ \\
\hline Ceftriaxone & $28(93.33 \%)$ & $1(3.33 \%)$ & $1(3.33 \%)$ & $9(100 \%)$ & $0(0 \%)$ & $0(0 \%)$ \\
\hline Norfloxacin & $29(96.67 \%)$ & $0(0 \%)$ & $1(3.33 \%)$ & $9(100 \%)$ & $0(0 \%)$ & $0(0 \%)$ \\
\hline Ciprofloxacin & $28(93.33 \%)$ & $0(0 \%)$ & $2(6.67 \%)$ & $8(88.89 \%)$ & $1(11.11 \%)$ & $0(0 \%)$ \\
\hline Nalidixic acid & $22(73.33 \%)$ & $7(23.33 \%)$ & $1(3.33 \%)$ & $8(88.89 \%)$ & $0(0 \%)$ & $1(11.11 \%)$ \\
\hline Chloramphenicol & $21(70 \%)$ & $7(23.3 \%)$ & $2(6.67 \%)$ & $7(77.78 \%)$ & $1(11.11 \%)$ & $1(11.11 \%)$ \\
\hline Gentamicin & $21(70 \%)$ & $6(20 \%)$ & $3(10 \%)$ & $7(77.78 \%)$ & $1(11.11 \%)$ & $1(11.11 \%)$ \\
\hline Amoxicillin & $0(0 \%)$ & $3(10 \%)$ & $27(90 \%)$ & $2(22.22 \%)$ & $0(0 \%)$ & $7(77.78 \%)$ \\
\hline
\end{tabular}

Parenthesis. $\mathrm{S}=$ sensitive; $\mathrm{I}$ = intermediate; $\mathrm{R}=$ resistance.

TABLE 3: Multidrug resistance pattern of Salmonella and Shigella species from diarrheal patients attending Nekemte Referral Hospital, October 2015 to February 2016.

\begin{tabular}{lcl}
\hline Bacterial Isolates & Number isolate & Antibiotics resisted \\
\hline Shigella species & $3(33.33 \%)$ & GEN, AM \\
\hline \multirow{3}{*}{ Salmonella species } & $1(3.33 \%)$ & C, AM \\
& $1(3.33 \%)$ & CIP, AM \\
& $1(3.33 \%)$ & C, CIP, CRO, NA, GEN, \\
\end{tabular}

Parenthesis. GEN = gentamicin; $\mathrm{AM}=$ amoxicillin; $\mathrm{C}=$ chloramphenicol; $\mathrm{CIP}=$ ciprofloxacin; $\mathrm{CRO}=$ ceftriaxone; $\mathrm{NA}=$ nalidixic acid; $\mathrm{NOR}=$ norfloxacin.

3.3. Antibiotic Susceptibility Pattern of the Isolates. Of all Salmonella isolates, $90 \%$ were resistant to amoxicillin $(30 \mu \mathrm{g})$ whereas only $10 \%, 6.7 \%, 6.7 \%, 3.3 \%, 3.3 \%$, and $3.3 \%$ of the isolates were found to be resistant to gentamicin $(10 \mu \mathrm{g})$, ciprofloxacin $(5 \mu \mathrm{g})$, chloramphenicol $(30 \mu \mathrm{g})$, ceftriaxone $(30 \mu \mathrm{g})$, norfloxacin $(10 \mu \mathrm{g})$, and nalidixic acid $(30 \mu \mathrm{g})$, respectively. Of all, $77.8 \%$ of Shigella isolates were resistant to amoxicillin, whereas $11.11 \%$ of them were found to be resistant to ciprofloxacin, chloramphenicol, gentamicin, and nalidixic acid. Shigella isolates were also found to be of high susceptibility to ceftriaxone and norfloxacin followed by ciprofloxacin, nalidixic acid, chloramphenicol, and gentamicin (Table 2). Multiple drug resistances were observed among Salmonella and Shigella isolates obtained from the participants of this study. Amazingly, one Salmonella isolate showed resistance to six antibiotics, while only $10 \%$ of the isolates exhibited multidrug resistance to different antibiotics tested in the study. Our results demonstrated that $33.33 \%$ of Shigella isolates exhibited multidrug resistance to gentamicin and amoxicillin (Table 3).

\section{Discussion}

According to this study, the predominant bacterial pathogens were Salmonella (7.11\%), indicating that it is common human health problem at the study area. The prevalence of Salmonella in this study is in agreement with the result (7.8\%) of study done in Bahir Dar [14] and higher than the result of studies done in Gonder (1.08\%) [8] and Addis Ababa 3.95\% [21], Ethiopia. Similarly, isolation rate of Salmonella species obtained in this study is higher than the results of studies in other countries, 3.3\% in Nigeria [11] and 1.5\% in Turkey [12]. In contrast, our result is lower than studies done in Gonder (17.39\%) [22], Harar (11.5\%) [13], Butajira (10.5\%) [23], and Jimma (10.8\%) [16], Ethiopia. The variation could be due to differences in sociodemographic characteristics, geographical location, and study time.

The isolation rate of Shigella species obtained in this study is lower than the earlier studies done in Ethiopia; 9.5\% in Bahir Dar [14], 9.1\% in Addis Ababa [21], 6.9\% in Mekelle [15], $6.7 \%$ in Harar [13], 4.57\% in Gonder [8], 4.5\% in Butajira [23], and $29 \%$ in Ambo [10]. Comparable results were reported by studies carried out elsewhere $2.9 \%$ in Nigeria Abeokuta [11] and 3\% in Turkey [12]. In contrast, the isolation rate of Shigella species in this study is higher than the results of studies done in Ambo (1.3\%) [24] and Jimma (1.1\%) [16].

Our results showed that $62.1 \%$ of the study participants wash their hands after using latrine. Good hand washing pattern after using latrine has statically significant association with prevalence of Salmonella and Shigella species. However, there were no statistically significant association between the percentage of patients with abdominal pain, joint pain, and prevalence of the pathogens. But there were statistically significant associations between headache, vomiting, and prevalence of Salmonella and Shigella species. The infection rate $(46.15 \%)$ within age group below 19 years might be due to difference in immunity status of the study group.

According to this study, the resistance rate of Salmonella was $90 \%$ to amoxicillin which is in agreement with reports from other areas of Ethiopia $[8,13,22,25]$. The possible reason could be due to long time service and wide use of this drug in the country and frequent exposure of Salmonella species to it. Salmonella species showed that less resistance to gentamicin in this study is lower than result (25\%) study done in Gonder [8]. In contrast, the result of this study is higher than results reported from other areas in Ethiopia [13, 21, 23].

On the other hand, Salmonella isolates showed lower $(3.33 \%)$ resistance rate to ceftriaxone and nalidixic acid than results obtained by earlier study done in Hawassa which were $75 \%$ and $25 \%$, respectively [25]. Similarly, resistance level of Salmonella isolates to chloramphenicol, gentamicin, and 
nalidixic acid is lower than results of studies conducted in Harar [13], Addis Ababa [21], and Gonder [8]. Resistance rate (3.33\%) of Salmonella isolates to ceftriaxone observed in this study is slightly higher than earlier studies done in other areas of the country $[8,21,23]$. The proportion $(10 \%)$ of multidrugresistant Salmonella isolates found in this study is lower than the previous reports [14].

Shigella species isolated in this study were highly susceptible to both ceftriaxone and norfloxacin (100\%) followed by ciprofloxacin and nalidixic acid. These findings are supported by the results of previous study conducted in other areas of Ethiopia [8, 21, 23]. The resistance rate Shigella isolates to amoxicillin was $77.78 \%$ which is in agreement with the study done in Gonder (88.2\%) [8] and slightly lower than result reported from Addis Ababa (91.4\%) [21], Harar (100\%) [13], and Hawassa (100\%) [25]. On the other hand, low resistant rate of Shigella isolates to chloramphenicol and gentamicin $(11.11 \%)$ observed in this study is contradictory to the results of earlier studies $[8,13,14,23,25]$ and supported by lower resistance level to gentamicin reported by other studies [11, 14, $21,23,26]$. More than $33 \%$ of Shigella isolates were multidrugresistant which is higher than the previous study [8] in Gonder, Ethiopia.

\section{Conclusion}

Our results revealed $9.2 \%$ prevalence of Salmonella than Shigella species among diarrheal patients in the study area. Antimicrobial susceptibility test results showed that Salmonella and Shigella species isolated during this study are highly resistant to amoxicillin. Salmonella and Shigella isolates were highly sensitive to ceftriaxone and norfloxacin. Based on the results of the current study we strongly recommend continuous surveillance on the prevalence and antibiotic resistance pattern of Salmonella and Shigella species among relevant patients in hospitals and other health centers in the study area which should be basis for empirical therapy. Since Salmonella and Shigella species were resistant to most common drugs, care should be taken in selecting antimicrobials in treating disease caused by them. Moreover, improving environmental and personal hygiene and providing safe potable water and intensive health education together with more careful use of antimicrobials could conserve antimicrobial efficacy and significantly reduce diarrheal illness.

In this study, the pathogens were not identified to the species level using serological analysis and hence biochemical tests should be substantiated by serological and molecular identification for better taxonomy of the pathogens to species and strain level. This study was conducted only on patients presented with diarrheal case and visited Nekemte Referral Hospital and did not include patients from health centers and private clinics. However, the study verified that further epidemiological study should be conducted on patients attending health centers and private clinics.

\section{Additional Points}

Availability of Data and Materials. Data supporting this research article are available from the corresponding author or first author on reasonable request.

\section{Conflicts of Interest}

There are no financial or other relationships that might lead to conflicts of interest in this study. The authors of this manuscript declare that they have no conflicts of interest.

\section{Authors' Contributions}

Mulissa Jida was involved in concept design, supervised the data collection and critical revision of the article, and provided final approval of the article. Alemayehu Terfassa was involved in concept design and data collection, drafted the manuscript, performed laboratory analysis, and provided analysis and interpretation of the data. All authors read and approved the final manuscript.

\section{Acknowledgments}

The authors are so grateful to Oromia Regional Health Laboratory, Nekemte, for providing laboratory materials, biochemical test reagents, and working bench, Nekemte Referral Hospital for assistance during stool sample collection, and Department of Biology, Wollega University, for supporting this research work.

\section{References}

[1] C. Yildiz, C. Öztürk, and G. Emekdas, "Research of the E. coli 0157:H7 strains cases of the Gastro-enterits," Infeksiyon Dergisi, vol. 19, pp. 189-192, 2005.

[2] M. A. Raji, S. F. H. Jiwa, M. U. Minga, and P. S. Gwakisa, "Escherichia coli 0157: H7 reservoir, transmission, diagnosis and the African situation: A review," East African Medical Journal, vol. 80, no. 5, pp. 271-276, 2003.

[3] M. Abdullahi, "Incidence and antimicrobial susceptibility pattern of Salmonella species in children attending some hospitals in kano metropolis, kano state-Nigeria," Bajopas, vol. 3, pp. 202-206, 2010.

[4] H. Yan, L. L. Alam, M. J. Shinoda, and S. Miyoshi, "Prevalence and antimicrobial resistance of Salmonella in retail foods in northern China," Food Microbiology, vol. 143, pp. 230-234, 2010.

[5] C. A. Arias and B. E. Murray, "Antibiotic-resistant bugs in the 21st century-a clinical super-challenge," The New England Journal of Medicine, vol. 360, no. 5, pp. 439-443, 2009.

[6] R. Sharma, C. L. Sharma, and B. Kapoor, "Antibacterial resistance: current problems and possible solutions," Indian Journal of Medical Sciences, vol. 59, no. 3, pp. 120-129, 2005.

[7] B. Gu, Y. Cao, S. Pan et al., "Comparison of the prevalence and changing resistance to nalidixic acid and ciprofloxacin of Shigella between Europe-America and Asia-Africa from 1998 to 2009," International Journal of Antimicrobial Agents, vol. 40, no. 1, pp. 9-17, 2012.

[8] T. A. Demissie, T. Moges, M. Feleke, M. Dagnachew, and A. Getnet, "Prevalence and antimicrobial susceptibility patterns of Shigella and Salmonella species among patients with diarrhea attending Gondar tOwn Health Institutions, Northwest Ethiopia," Science Journal of Public Health, vol. 2, no. 5, pp. 469475, 2014.

[9] K. Huruy, A. Kassu, A. Mulu et al., "High level of antimicrobial resistance in Shigella species isolated from diarrhoeal patients 
in University of Gondar Teaching Hospital, Gondar, Ethiopia," Pharmacologyonline, vol. 2, pp. 328-340, 2008.

[10] S. Kefyalew, G. Kebede, and A. Keneni, "Prevalence of Shigella related diarrhea in Ambo town and antibiotic susceptibility of the isolated strains," Greener Journal of Epidemiology and Public Health, vol. 3, no. 1, pp. 001-006, 2015.

[11] O. A. Akingbade, P. O. Okerentugba, O. B. Awoderu, and A. A. Shobayo, "Prevalence of Samonella sp and Shigella $s p$ among children with diarrhoea in Abeokuta, Ogun State, Nigeria," Academ Arena, vol. 6, no. 9, pp. 13-16, 2014.

[12] T. T. Kara, H. Özdemir, F. Kurt et al., "Prevalence of Salmonella and Shigella spp. and antibiotic resistance status in acute childhood gastroenteritis, Ankara, Turkey," J. Pediatr. Inf., vol. 9, pp. 102-107, 2015.

[13] A. A. Reda, B. Seyoum, J. Yimam, G. Andualem, S. Fiseha, and J. M. Vandeweerd, "Antibiotic susceptibility patterns of Salmonella and Shigella isolates in Harer, Eastern Ethiopia," Journal of Infectious Diseases and Immunity, vol. 3, no. 8, pp. 134-139, 2011.

[14] M. A. Admassu, G. Yemane, M. Kibret, B. Abera, E. Nibret, and M. Adal, "Prevalence and antibiogram of Shigella and Salmonella spp. from under five children with acute diarrhea in Bahir Dar Town," Ethiopian Journal of Science and Technology, vol. 8, no. 1, pp. 27-35, 2015.

[15] A. Gebrekidan, T. A. Dejene, G. Kahsay, and A. G. Wasihun, "Prevalence and antimicrobial susceptibility patterns of Shigella among acute diarrheal outpatients in Mekelle hospital, Northern Ethiopia," BMC Research Notes, vol. 8, no. 611, 2015.

[16] T. Lamboro, T. Ketema, and K. Bacha, "Prevalence and antimicrobial resistance in Salmonella and Shigella species isolated from outpatients, Jimma University Specialized Hospital, Southwest Ethiopia," Canadian Journal of Infectious Diseases \& Medical Microbiology, vol. 2016, Article ID 4210760, 8 pages, 2016.

[17] W. W. Daniel, Biostatistics, A Foundation for Analysis in The Health Sciences, John Wiley and Sons, New York, USA, 7th edition, 1999.

[18] WHO, Basic Laboratory Procedure in Clinical Bacteriology, 5669, World Health Organization, Geneva, 2nd edition, 2003.

[19] M. Cheesbrough, Medical Laboratory Manual for Tropical Countries, vol. 2, 38-39, 138, UK, Cambridge Press, 2nd edition, 2006.

[20] CLSI, Performance Standard for Antimicrobial Susceptibility Testing, Wayne, PA 19087, 2012.

[21] Y. Mamuye, G. Metaferia, A. Birhanu, K. Desta, and S. Fantaw, "Isolation and antibiotic susceptibility patterns of Shigella and Salmonella among under 5 children with acute diarrhoea: a cross-sectional study at selected public health facilities in addis ababa, ethiopia," Clinical Microbiology: Open Access, vol. 4, no. 186, 2015.

[22] L. Garedew, Z. Hagos, Z. Addis, R. Tesfaye, and B. Zegeye, "Prevalence and antimicrobial susceptibility patterns of Salmonella isolates in association with hygienic status from butcher shops in Gondar town, Ethiopia," Antimicrobial Resistance and Infection Control, vol. 4, no. 1, article 21, 2015.

[23] G. Mengistu, G. Mulugeta, T. Lema, and A. Aseffa, "Prevalence and antimicrobial susceptibility patterns of Salmonellaserovars and Shigella species," Journal of Microbial \& Biochemical Technology, vol. 6, Supplemet 2, Article ID Article 6, 2014.

[24] W. Tosisa, Prevalence And Antibiotic Resistance of Enteric Bacterial Pathogens Isolated from Childhood Diarrhea in Ambo Town Public Health Institutions, Addis Ababa University, 2015.
[25] M. Getamesay, B. Getenet, and Z. Ahmed, "Prevalence of Shigella, Salmonella and Cmpylobacter species and their susceptibility patters among under five children with diarrhea in Hawassa Town, South Ethiopia," Ethiopian Journal of Health Sciences, vol. 24, no. 2, pp. 101-108, 2014.

[26] G. Yismaw, C. Negeri, and A. Kassu, "A five-year antimicrobial resistance pattern observed in Shigella species isolated from stool samples in Gondar University Hospital, northwest Ethiopia," The Ethiopian Journal of Health Development, vol. 20, no. 3, pp. 194-198, 2006. 


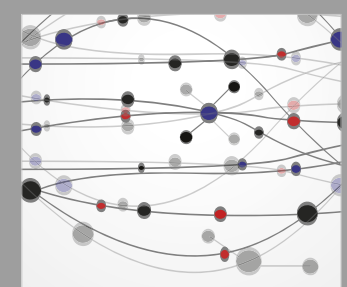

The Scientific World Journal
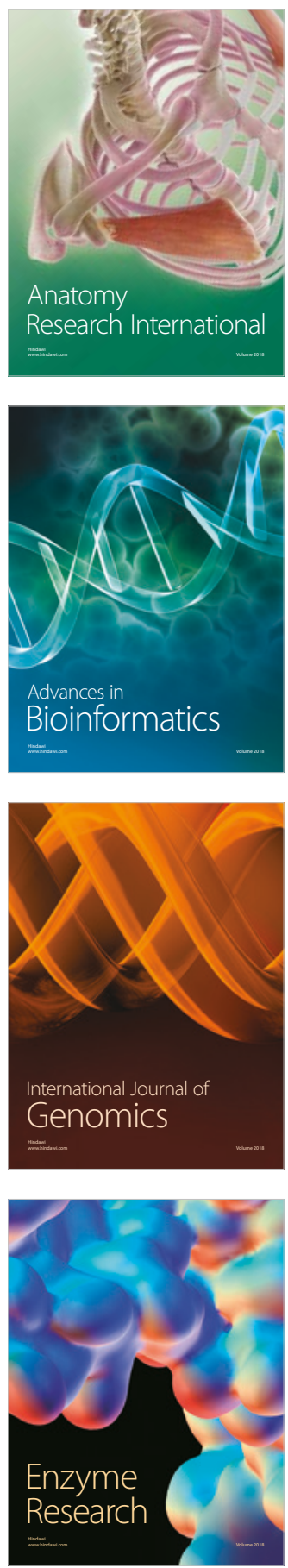
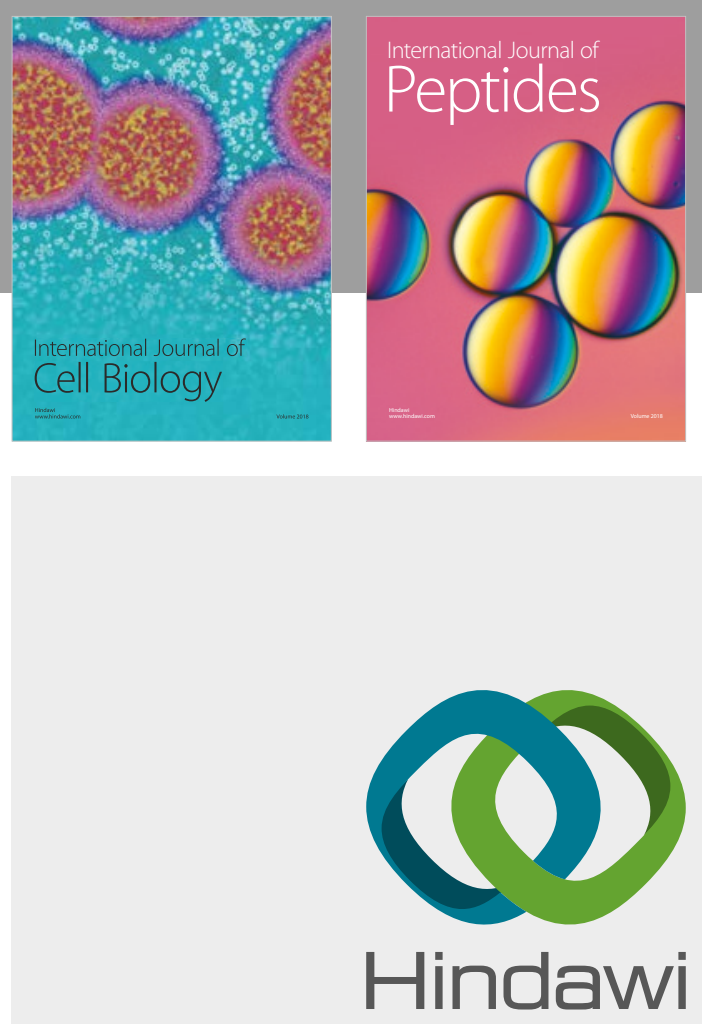

Submit your manuscripts at

www.hindawi.com
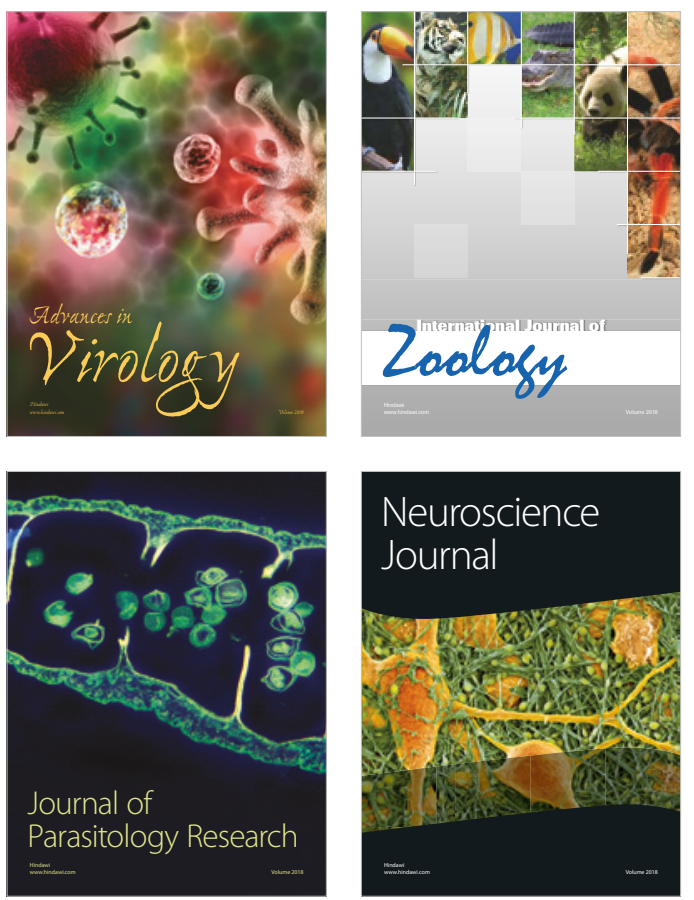
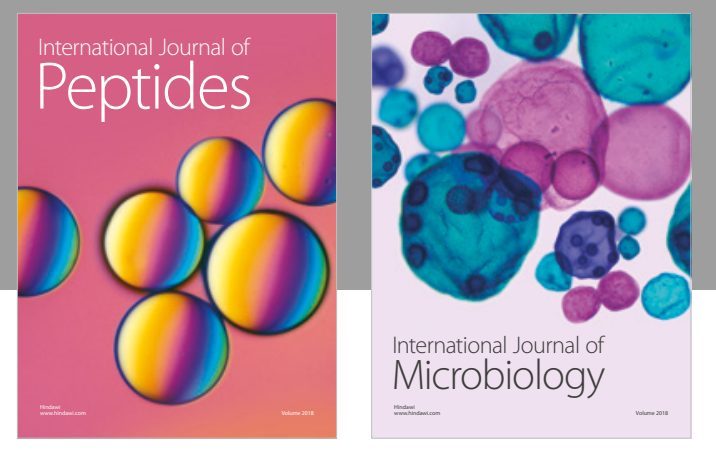

nternational Journal of Microbiology
Journal of
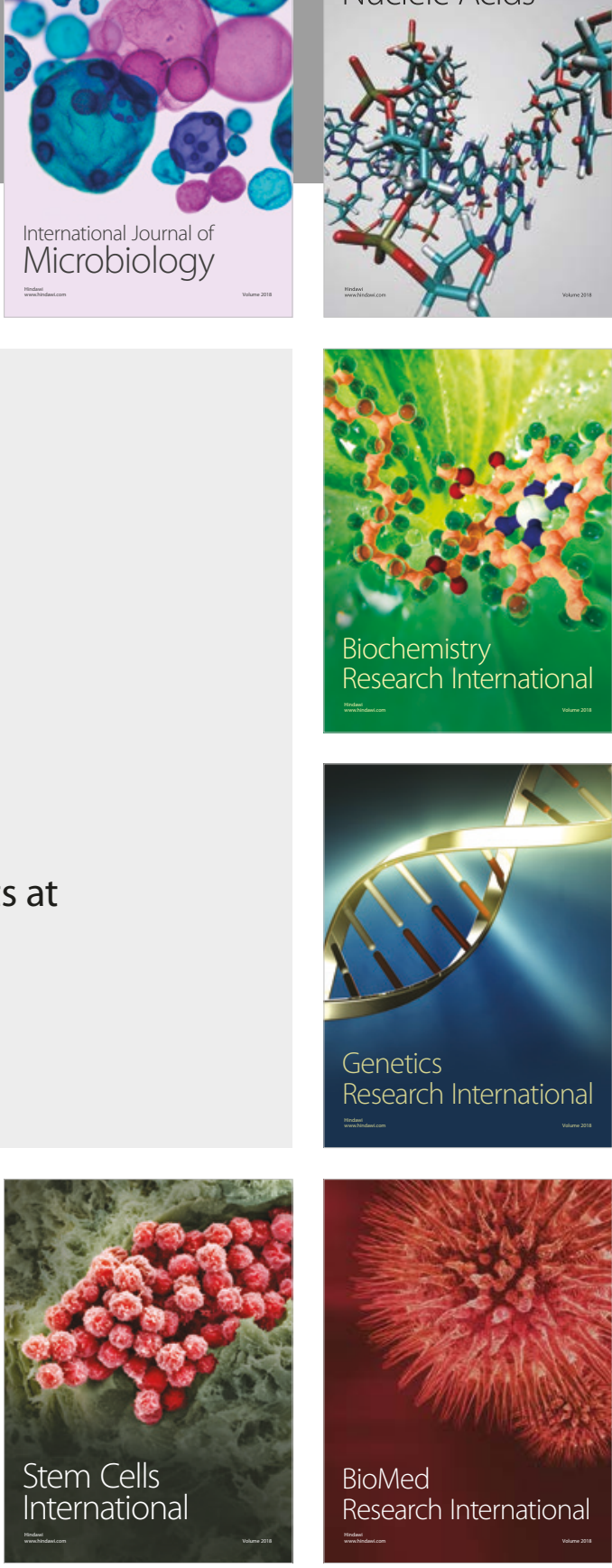
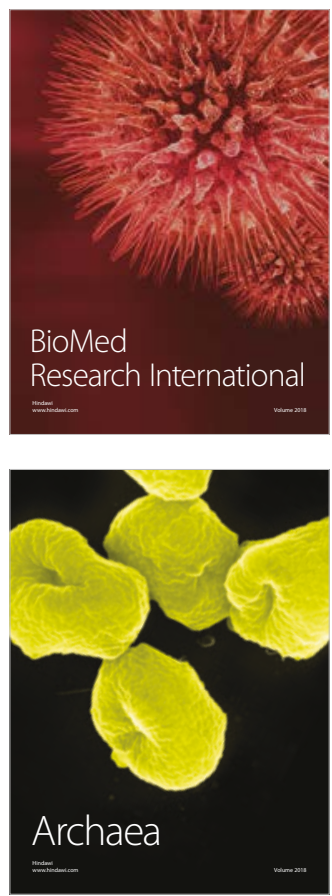